\title{
Surgical strategy for temporal lobe epilepsy with dual pathology and incomplete evidence from EEG and neuroimaging
}

\author{
LAN LUAN $^{1}$, YUQIANG SUN ${ }^{2}$ and KANG YANG ${ }^{1}$ \\ ${ }^{1}$ Department of Neurosurgery, The Second Hospital of Dalian Medical University, Dalian, Liaoning 116000; \\ ${ }^{2}$ Department of Neurosurgery, The Central Hospital of Harbin, Harbin, Heilongjiang 150000, P.R. China
}

Received February 22, 2018; Accepted August 2, 2018

DOI: $10.3892 / e t m .2018 .6774$

\begin{abstract}
Coexistence of hippocampal sclerosis (HS) and a temporal neocortical lesion, including focal cortical dysplasia, vascular malformations or benign primary brain tumors, is defined as dual pathology. In the majority of cases, the complete evidence based on electroencephalogram (EEG) and magnetic resonance imaging (MRI) for each of the dual pathological lesions is difficult to obtain. As a result, patients with dual pathology are poor surgical candidates due to potential incomplete resection of the epileptogenic zone. The current study retrospectively reviewed 24 patients with potential dual pathology and incomplete EEG and MRI evidence, aiming to provide novel surgical strategies. The results revealed a significantly worse seizure-free outcome for patients with incomplete EEG and MRI data compared with patients who received a clear diagnosis of dual pathology based on complete test results (11.1 vs. 60.0\%; $\mathrm{P}<0.05)$. Notably, satisfying surgical (seizure-free) outcomes were still achieved in $60 \%$ of patients with unclear EEGs to locate the onset zone and clear MRI scans identifying the sclerotic hippocampus and nearby neocortical lesions, which could be resected together. In patients with multiple epileptogenic onsets or with widespread epileptic discharges, the hippocampus should be spared from resection due to concern for potential postsurgical mood or memory disorders. In conclusion, patients with resection of the sclerotic hippocampus and neocortical lesion had improved seizure-free outcomes compared with patients who received individual lesion resection. The current study aimed to provide a novel surgical strategy for neurosurgeons treating epileptic patients with dual pathology.
\end{abstract}

Correspondence to: Dr Kang Yang, Department of Neurosurgery, The Second Hospital of Dalian Medical University, 467 Zhongshan Road, Dalian, Liaoning 116000, P.R. China

E-mail: dmu_yk@126.com

Key words: dual pathology, epilepsy, hippocampal sclerosis, focal cortical dysplasia

\section{Introduction}

Temporal lobe epilepsy (TLE) is the most common form of partial epilepsy in humans (40-60\%) and in $~ 30 \%$ of patients it is refractory to treatment and drug resistance emerges (1). A randomized clinical trial has demonstrated that surgery is superior to prolonged drug treatment for patients with refractory TLE (2). However, surgical treatment remains unsuccessful in providing a seizure-free outcome in $20-30 \%$ of patients with TLE (3). The coexistence of hippocampal sclerosis (HS) and temporal neocortical lesions, including focal cortical dysplasia (FCD), vascular malformations and benign primary brain tumors, is termed dual pathology, which may be a major cause of surgical failure in patients with TLE (4-7).

A majority of epileptic cases with dual pathology are poor surgical candidates due to unclear diagnosis leading to failure of complete resection of the epileptogenic zone (5). Clear and complete electroencephalogram (EEG) and magnetic resonance imaging (MRI) evidence for each dual pathological lesion is rare. Of note, EEG and MRI-based diagnosis of one epileptogenic lesion typically guides the diagnosis of other lesions (8). Several studies have indicated that the surgical outcome for patients with neocortical epilepsy were less satisfactory compared with patients with mesial temporal lobe epilepsy (mTLE), a type of epilepsy originates from mesial temporal lobe structure, including the hippocampus and amygdala (9-12). HS is the most common underlying pathological cause of mTLE ( $60-80 \%)$, and in a majority of cases, MRI findings of unilateral HS predict a favorable prognosis $(13,14)$. Additionally, several reports have clarified that neocortical lesions, including FCD, are associated with early seizure onset, high seizure frequency and poor postsurgical outcomes $(4,15,16)$. Resection of the mesial temporal lobe instead of the neocortical lesion in patients with dual pathology is therefore favored, particularly when MTLE foci has complete EEG and MRI evidence (8). In other cases, surgical decisions are more difficult.

The present study investigated the surgical outcome for patients with temporal lobe dual pathology in the absence of matched EEG and MRI findings. While surgical indication of resecting both lesions is not sufficient, prognostic implications of the severity of pathology, clinical factors, surgical procedures and the results of presurgical diagnostic modalities were examined, aiming to provide a novel surgical strategy for similar cases. 


\section{Materials and methods}

Patients. Data of 24 patients with TLE and dual pathology presurgically verified by radiologic and EEG evidence was reviewed retrospectively. A total of 14 females and 10 males participated in the study (mean age, 26.0 \pm 8.5 years). Patients were surgically treated at the Epilepsy Center of Liao Ning Province, The Second Hospital of Dalian Medical University (Liaoning, China) between January 2012 and December 2015. Selected patients exhibited a coexistence of HS and a temporal neocortical lesion, with either radiologic or EEG proof. Patients with other causes of seizure, including brain trauma, encephalitis and meningitis, or diagnosed with idiopathic epilepsy were excluded. Clinical features, including age at onset, age at surgery, duration of epilepsy, seizure frequency, preoperational EEG and radiologic data, surgical strategy, postsurgical pathological diagnosis and outcome were examined (Table I). All patients were followed-up for $\geq 2$ years. The present study was approved by the Ethics Committee of The Second Hospital of Dalian Medical University (Liaoning, China).

MRI. All patients underwent a 3.0-T cerebral MRI scan (MAGNETOM Skyra, Siemens Healthineers, Erlangen, Germany). MRI sequences included T1-weighted and T2-weighted axial, sagittal and coronal images at a section thickness of $1.5 \mathrm{~mm}$. T2-weighted fluid-attenuated inversion recovery sequences were further obtained at an angle perpendicular to the long axis of the hippocampus.

EEG monitoring. Long-term scalp V-EEG was performed with additional anterior temporal electrodes placed on the scalp of patients. A total of 13 patients did not receive a conclusive diagnosis from V-EEG. Therefore, electrocorticography was performed in these patients with a combination of subdural grids and strips on the cortex of the patient. Intracranial electrodes were installed in various combinations, always directed from the lateral neocortex to the para-hippocampal gyrus, to monitor mesial temporal epileptiform discharges, enabling to locate the epileptogenic onset. For patients with FCD or a temporal tumor identified by MRI scans, and an unclear on-scalp EEG, subdural electrodes were placed over the area of the lesion. A localizing pattern of ictal onset/interictal spike was defined by monitoring the electrodes of an epileptogenic lobe or two adjacent electrodes.

Groups and surgical strategies. Based on presurgical evaluations, including clinical symptoms, MRI scans and EEG results, groups were divided and surgical strategies and resection margins were defined accordingly (Table II). In group 1, HS was confirmed by MRI and EEG and a temporal lesion was detected by MRI but not by EEG. An anterior temporal lobectomy and the resection of the temporal neocortical lesion were performed. Anterior temporal lobectomy was defined as the removal of medial structures, including the amygdala, hippocampus and parahippocampal gyrus. In group 2, HS was detected by MRI. EEG revealed epileptic discharges in the anterior temporal lobe (ATL) and was unable to locate the onset zone to the hippocampus without depth electrodes. A temporal lesion around the hippocampus was detected by MRI. The surgical strategy was to resect the neocortical lesion and the hippocampus to completely remove the potential epileptogenic focus. In group 3, HS was detected by MRI, with multiple epileptic onset or widespread epileptic discharges detected on the EEG. A temporal lesion was detected by MRI. A resection of the temporal lesion was performed and ATL, including the hippocampus, was spared. In group 4, HS was unclear on the MRI, but the epileptic onset was captured by the EEG. A distinct temporal lesion was detected by MRI. The surgical strategy was to resect the temporal lesion, following a discussion with and consensus from the patients.

Notably, there was a concern that the resection of the bilateral hippocampus may be associated with unexpected memory and emotional dysfunction following surgery, which may be considered as postoperative complication. Therefore, only with complete MRI and EEG evidence confirming that the hippocampus was responsible for the epileptic onset was the hippocampus resected.

Neuropathological examination. Postsurgical pathological diagnoses of temporal lesions, including vascular malformations and benign primary brain tumors, were performed according to the WHO classification of tumors of the central nervous system (17). Diagnosis of the severity and classification of FCD was based on standards described by Palmini et al (18) (Table III).

Postoperative follow-up. Seizure-free was defined as patients receiving antiepileptic treatments with no seizures and EEG abnormalities for $\geq 2$ years (4). All patients were followed-up for 24-36 months. Follow-up data were collected annually in the subsequent years and included information regarding epilepsy attacks, antiepileptic drugs taken, neuroimaging and EEG monitoring. The mean follow-up duration was $30.7 \pm 1.8$ months and median follow-up time was 33 months (range, 24-36 months). Surgical outcome was classified according to the Engel classification (4): (Ia) Completely seizure-free; (I) seizure-free, or auras or convulsions with drug withdrawal only; (II) rare seizures ( $<2$ seizures/year or $>90 \%$ seizure reduction); (III) reduction of seizure frequency $>75 \%$; and (IV) reduction of seizure frequency $<75 \%$.

Statistical tests. All statistical analyses, including the clinical characteristics of the seizure-free group with the non-seizure-free group, were performed using the $\chi^{2}$ test. All analyses were conducted using SPSS version 12.0 (SPSS, Inc., Chicago, IL, USA) and STATA version 9.2 (Stata Corp LP, College Station, TX, USA). $\mathrm{P}<0.05$ was considered to indicate a statistically significant difference.

\section{Results}

Patient characteristics and surgical outcomes. Between January 2012 and December 2015, a total of 24 patients were diagnosed and underwent surgery for dual pathology due to HS and temporal lesions, including FCD or benign tumors, at the Epilepsy Center of Liao Ning Province, The Second Hospital of Dalian Medical University (Liaoning, China). Patients were followed-up for 24-36 months postoperatively and all data were analyzed retrospectively. Among the patients were $14 / 24(58.3 \%)$ females and 10/24 (41.7\%) were males, with a mean age of $26.0 \pm 8.5$ years (range, 17-51 years) at time of 
Table I. Clinical characteristics of 24 patients with dual pathology diagnosis.

\begin{tabular}{|c|c|c|c|c|c|c|}
\hline Sex & $\begin{array}{l}\text { Age of onset } \\
\text { (years) }\end{array}$ & $\begin{array}{c}\text { Duration of } \\
\text { epilepsy (years) }\end{array}$ & $\begin{array}{c}\text { MRI } \\
\text { findings }\end{array}$ & $\begin{array}{c}\text { EEG } \\
\text { findings }\end{array}$ & $\begin{array}{l}\text { Pathological } \\
\text { findings }\end{array}$ & $\begin{array}{c}\text { Engel } \\
\text { classification }\end{array}$ \\
\hline Male & $<1$ & 50 & $\mathrm{HS}+\mathrm{FCD}$ & mTLE+FO & $\mathrm{HS}+\mathrm{FCD}$ & I \\
\hline Female & 6 & 18 & $\mathrm{HS}+\mathrm{VM}$ & mTLE+FO & $\mathrm{HS}+\mathrm{VM}$ & II \\
\hline Male & 18 & 22 & $\mathrm{HS}+\mathrm{FCD}$ & mTLE & $\mathrm{HS}+\mathrm{FCD}$ & I \\
\hline Male & 13 & 7 & $\mathrm{HS}+\mathrm{FCD}$ & mTLE & $\mathrm{HS}+\mathrm{FCD}$ & II \\
\hline Female & 15 & 2 & HS+tumor & mTLE & HS+tumor & II \\
\hline Female & 12 & 22 & $\mathrm{HS}+\mathrm{FCD}$ & mTLE & $\mathrm{HS}+\mathrm{FCD}$ & I \\
\hline Female & 8 & 17 & $\mathrm{HS}+\mathrm{FCD}$ & mTLE+FO & $\mathrm{HS}+\mathrm{FCD}$ & I \\
\hline Male & 17 & 15 & $\mathrm{HS}+\mathrm{FCD}$ & mTLE+FO & $\mathrm{HS}+\mathrm{FCD}$ & I \\
\hline Male & 14 & 24 & HS+tumor & mTLE & HS+tumor & I \\
\hline Female & 7 & 11 & $\mathrm{HS}+\mathrm{FCD}$ & mTLE & $\mathrm{HS}+\mathrm{FCD}$ & II \\
\hline Male & 11 & 6 & $\mathrm{HS}+\mathrm{FCD}$ & $\mathrm{NC}$ & $\mathrm{HS}+\mathrm{FCD}$ & II \\
\hline Female & 13 & 10 & $\mathrm{HS}+\mathrm{FCD}$ & $\mathrm{FO}$ & $\mathrm{HS}+\mathrm{FCD}$ & I \\
\hline Female & 23 & 21 & $\mathrm{HS}+\mathrm{VM}$ & $\mathrm{NC}$ & $\mathrm{HS}+\mathrm{VM}$ & I \\
\hline Male & 15 & 20 & $\mathrm{HS}+\mathrm{FCD}$ & $\mathrm{FO}$ & $\mathrm{HS}+\mathrm{FCD}$ & II \\
\hline Male & 19 & 16 & $\mathrm{HS}+\mathrm{FCD}$ & $\mathrm{NC}$ & $\mathrm{HS}+\mathrm{FCD}$ & I \\
\hline Male & 22 & 22 & $\mathrm{HS}+\mathrm{VM}$ & $\mathrm{N}$ & VM & II \\
\hline Female & 18 & 9 & $\mathrm{HS}+\mathrm{FCD}$ & $\mathrm{N}$ & FCD & II \\
\hline Male & 11 & 14 & $\mathrm{HS}+\mathrm{FCD}$ & Wide-spread discharges & FCD & III \\
\hline Female & 17 & 21 & HS+tumor & $\mathrm{N}$ & Tumor & I \\
\hline Female & 11 & 17 & $\mathrm{HS}+\mathrm{FCD}$ & Wide-spread discharges & FCD & II \\
\hline Female & 6 & 19 & $\mathrm{HS}+\mathrm{FCD}$ & Wide-spread discharges & FCD & III \\
\hline Female & 8 & 20 & VM & mTLE & VM & II \\
\hline Female & 10 & 21 & FCD & mTLE & FCD & III \\
\hline Female & 9 & 25 & Tumor & $\mathrm{mTLE}+\mathrm{FO}$ & Tumor & II \\
\hline
\end{tabular}

HS, hippocampal sclerosis; FO, focal onset of temporal neocortex; mTLE, mesial temporal lobe epilepsy; FCD, focal cortical dysplasia; VM, vascular malformation; $\mathrm{NC}$, unclear of epileptic onset; $\mathrm{N}$, no ictal onset.

surgery. Mean age at seizure onset was $12.7 \pm 1.1$ years (range, $<1-23$ years) and the mean interval between seizure onset and surgery was $17.8 \pm 1.9$ years (range, $2-50$ years).

According to the postsurgical follow-up data, 10/24 (41.7\%) patients were seizure-free, while 14/24 (58.3\%) patients did not achieve this outcome.

Typical MRI findings of dual pathology lesions, including hippocampal sclerosis and neocortical malformation, were located in the right temporal lobe (in Fig. 1) and multiple continuous slowing epileptic discharges from the left anterior temporal electrodes, indicating a potential onset zone located in hippocampus, are presented in the EEG (Fig. 2).

Analysis of surgical outcomes based on patient characteristics. Data representing the surgical outcomes and patient characteristics were analyzed and suggested that the male/female ratio, age of onset, duration of epilepsy and seizure frequency were not associated with postsurgical outcome (Table IV).

Pathological diagnosis revealed 16/24 (66.7\%) cases of FCD. Hippocampal resection was performed in 15/24 (62.5\%) patients, which was considered to be associated with a higher chance of becoming seizure-free (44\% vs. $60 \%$ for FCD vs. hippocampal resection). Pathological findings of temporal lobe lesions are presented as hippocampal sclerosis (Fig. 3A), FCD (Fig. 3B) and ganglioma (Fig. 3C).

A complete resection of the potential epileptogenic area was performed in $15 / 24(62.5 \%)$ patients. A total of $9 / 24$ $(37.5 \%)$ patients did not receive complete resection and the hippocampus was spared due to a lack of either MRI abnormality or corresponding ictal discharges in the EEG.

Statistics of surgical strategy and outcomes. According to the presurgical evaluation, patients were divided into four groups (Table II). Patients of group 1, with explicit evidence on MRI and EEG for HS and another temporal lesion on MRI scan, received complete resection of the abnormalities and exhibited a significantly better outcome compared with groups $3(60.0 \%$ vs. $11.1 \% ; \mathrm{P}=0.03)$ and $4(60.0 \%$ vs. $0 \%$; $\mathrm{P}=0.01$; Table V). Patients of group 2 further had a significantly better chance to become seizure-free compared with group 3 (60.0\% vs. $11.1 \% ; \mathrm{P}=0.03)$ and $4(60.0 \%$ vs. $0 \% ; \mathrm{P}=0.01)$, which may be associated with the resection of an increased amount of the potential epileptogenic area. The association of the surgical strategy and patients classed as groups 3 and 4 was not determined due to the small sample numbers. However, it was 
Table II. Pathology of resection-based grouping.

\section{A, Group 1}

Variable

Hippocampal sclerosis

Temporal lesion

Analytical method

MRI

EEG

Surgical strategy

\section{B, Group 2}

Variable

Analytical method

MRI

EEG

Surgical strategy

C, Group 3
Hippocampal sclerosis

Temporal lesion

\author{
NC (limited to ATL)
}

$+$

N

Variable

Hippocampal sclerosis

Temporal lesion

Analytical method

MRI

$+$

EEG

NC (multiple onsets or wide-spread discharges)

$+$

Surgical strategy

Resection of the temporal lesion

D, Group 4

Variable

Hippocampal sclerosis

Temporal lesion

Analytical method

MRI

EEG

Surgical strategy

$\mathrm{N}+$

Resection of the temporal lesion

$\mathrm{NC}$, unclear of epileptic onset; $\mathrm{N}$, no ictal onset.

Table III. Diagnosis criteria of FCD according to Palmini's classification.

Classification

mMCD I

mMCD II

FCD 1A

FCD 1B

FCD 2A

FCD 2B
Pathologic findings

Ectopically placed neurons in cortex molecular layer only Ectopically placed neurons outside of cortex molecular layer only Isolated architectural abnormalities (dyslamination)

Additional immature or giant neurons

Presence of dysmorphic neurons

Additional balloon cells

mMCD, mild malformation of cortical development; FCD, focal cortical dysplasia.

suggested that group 3 had a significantly increased chance of becoming seizure-free compared with group 4 (16.7\% vs. $0 \%$;
$\mathrm{P}=0.02)$. Furthermore, EEG findings of epileptic discharges may be important for the decision of resecting, considering 

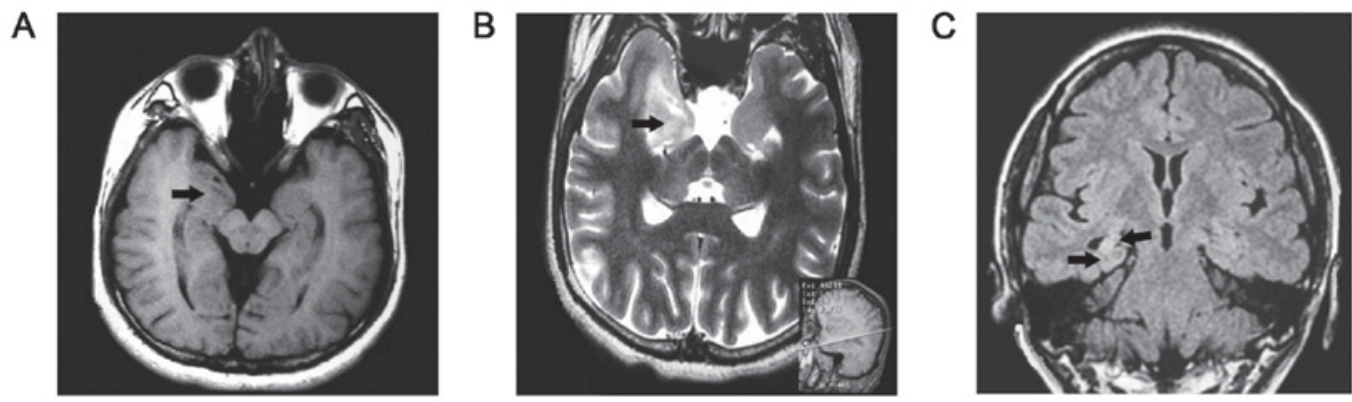

Figure 1. Typical MRI findings of lesions. (A) A T1-weighted MRI scan highlighting a neocortex malformation of the right anterior temporal lobe (arrow). (B) A T2-weighted MRI scan reveals a lesion in the right mesial temporal lobe, which pathology confirmed as ganglioglioma, a benign brain tumor (indicated by the arrow). A reference line parallel to the canthomeatal line within the brain is presented in the bottom right (sagittal view). (C) A fluid-attenuated inversion recovery T2-weighted MRI scan reveals a reduced hippocampal volume with increased signal in the right mesial temporal structures and slightly enlarged temporal horns (arrow); typical findings of hippocampal sclerosis. MRI, magnetic resonance imaging.

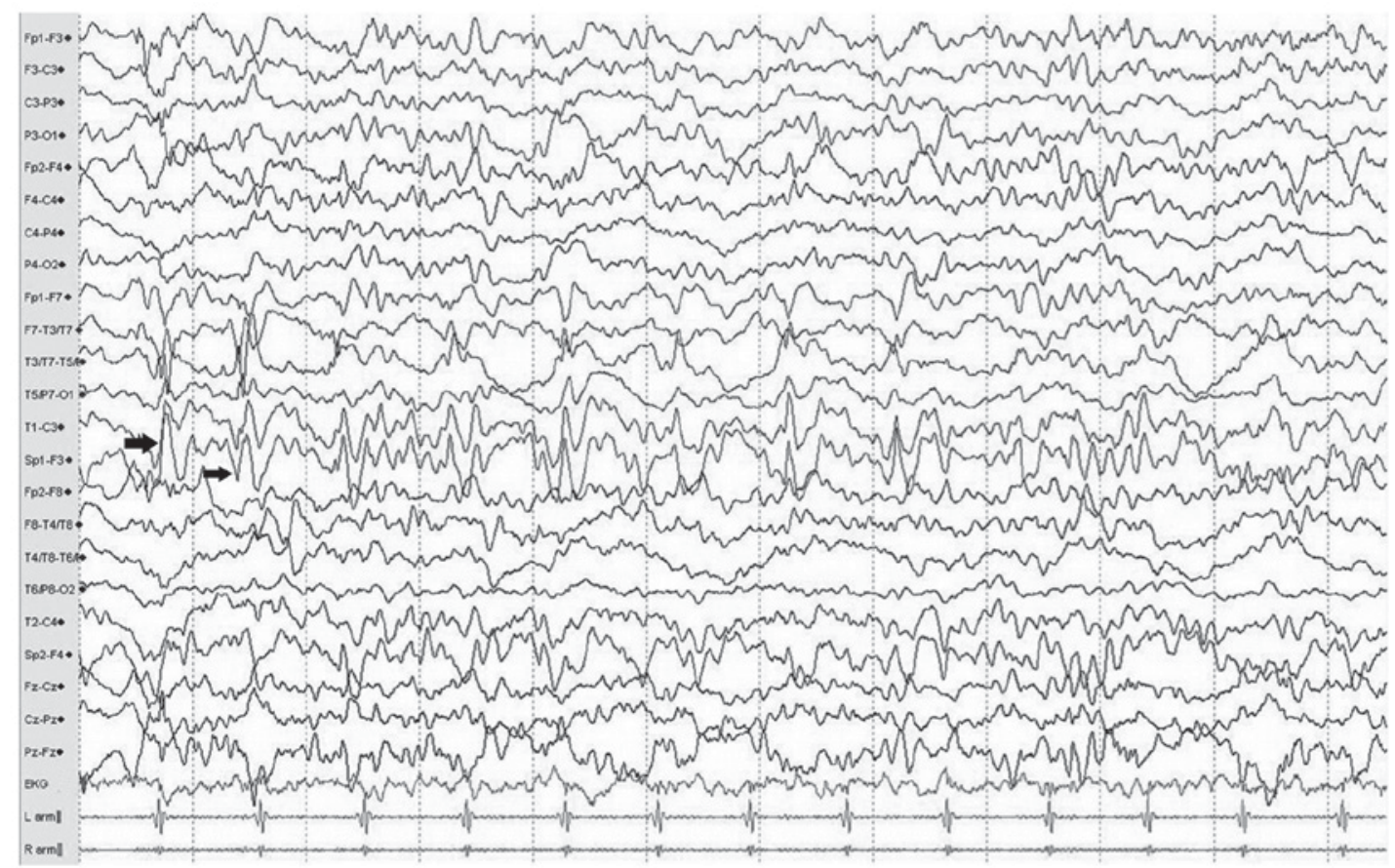

Figure 2. Typical electroencephalogram of a patient with hippocampal sclerosis. Traces exhibit multiple continuous slowing epileptic discharges (indicated with arrows) from the left anterior temporal electrodes (Sp1 and T1) during the interictal period, which indicate a potential onset zone located in the mesial or lateral anterior temporal lobe.

that patients of group 3 exhibited EEG abnormalities while patients of group 4 did not.

\section{Discussion}

HS is frequently associated with neocortical lesions, including FCD (5). Coexistence of HS with another lesion is a well-known phenomenon that is defined as dual pathology (8). There are various explanations for the pathogenesis of dual pathology, including the secondary development of HS from seizures induced by FCD or seizures resulting from the combined effect of HS and FCD (19-22). Another study suggested that according to EEG findings, there was no epileptic discharge spreading between the hippocampus and the extra-temporal lobe, which may indicate an association between the pathogenic mechanisms during embryogenesis and early development instead of epileptic pathways between them (23). Regarding therapy, it has been widely accepted that surgery is a beneficial solution for patients with epilepsy and dual pathology (8). However, the demarcation of resection and the association with or independence from epilepsy is still debated (11). Salanova et al (6) claimed that the complete resection of the dual pathology lesions was associated with an improved outcome. A total of $70.2 \%$ of the patients with complete resection were seizure-free and $21.6 \%$ had rare seizure attacks; $30-60 \%$ of patients with TLE achieved Engel class I (1). Patients that only received a hippocampal resection suffered from frequent seizure attacks and are described as Engel class III and IV (6). Li et al (24) published a study that included 38 patients with dual pathology and 3 patients that underwent two surgical procedures. Based on the location of resection, the outcome of three groups, defined as simple lesion resection, hippocampus resection and dual pathology complete resection, were compared. Following a 37-month 
Table IV. Analysis of surgical outcomes based on patient characteristics.

\begin{tabular}{|c|c|c|c|}
\hline Variable & Seizure-free & Not seizure-free & P-value \\
\hline Male/female & $5 / 5$ & $5 / 9$ & 0.21 \\
\hline Age of onset (years) & $13.5 \pm 1.7$ & $11.6 \pm 1.3$ & 0.25 \\
\hline Duration of epilepsy (years) & $21.9 \pm 3.4$ & $15.0 \pm 1.8$ & 0.18 \\
\hline Resection of FCD & $7 / 16(43.7 \%)$ & $9 / 16(56.0 \%)$ & 0.12 \\
\hline Resection of HS & $9 / 15(60.0 \%)$ & $6 / 15(40.0 \%)$ & 0.04 \\
\hline Complete resection of dual pathology & $9 / 15(60.0 \%)$ & $6 / 15(40.0 \%)$ & 0.04 \\
\hline
\end{tabular}

Data are presented as $\mathrm{n}(\%)$ or mean \pm standard deviation. FCD, focal cortical dysplasia; HS, hippocampal sclerosis.

Table V. Statistics of surgical strategy and outcomes.

\begin{tabular}{lccc}
\hline Surgical strategy & Cases (n) & Seizure-free (n) & Seizure-free (\%) \\
\hline Dual pathology resection & 15 & 9 & 60.0 \\
Group 1 & 10 & 6 & 60.0 \\
Group 2 & 5 & 3 & 60.0 \\
Temporal lesion resection & 9 & 1 & 11.1 \\
Group 3 & 6 & 1 & 16.7 \\
Group 4 & 3 & 0 & 0 \\
\hline
\end{tabular}
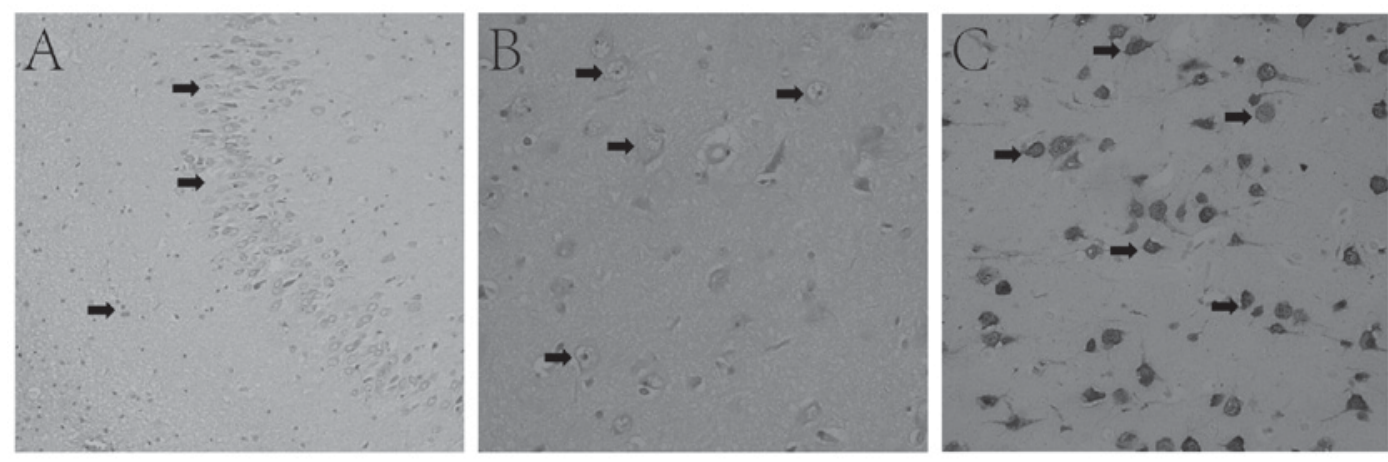

Figure 3. Typical pathological findings of lesions in patients with HS. (A) H\&E staining. Arrows highlight dispersed granular cells and decreased neurons and increased glial cells in the hippocampus dentate gyrus, indicative of HS (magnification, x20). (B) H\&E staining of FCD in the anterior temporal lobe presents a disorganized cortex, giant neurons with disordered polarity and dysmorphic neurons (arrows), indicating FCD IIA (magnification, x40). (C) Immunohistochemical staining of cluster of differentiation 34 expressed in vessels and ganglion cells (arrows) confirming temporal ganglioma (magnification, x40). HS, hippocampal sclerosis; H\&E, hematoxylin and eosin; FCD, focal cortical dysplasia.

follow-up, outcome analysis reported $12.5,20.0$ and $73.0 \%$ of patients, respectively, as seizure-free.

In the present study, 15 patients underwent complete resection of dual pathology and 9 of these patients were seizure-free $(60 \%)$ post surgery, which was similar to previous reports $(6,22)$. It was further demonstrated that improved outcomes may be achieved when patients receive a complete resection of the neocortical lesion and HS compared with the resection of the neocortical lesion alone (60.0 vs. 11.1\%).

The sensitivity of MRI scans and EEG examinations remained problematic as these features hold the key for the diagnosis of dual pathology and establishing a surgical strategy is usually difficult as a result of incomplete evidence for both lesions. The hippocampus may likely be associated with epilepsy and is the preferred target of resection for surgeons. The present study suggested that, compared with patients who had EEG and MRI-based proof for dual pathology, a satisfactory surgical outcome, classification as Engel class I, was still achieved in patients whose EEG diagnosis was not convincing, yet their dysplastic tissue and hippocampus were still removed together (Group 1 vs. 2; 60\% vs. 60\%). This outcome was based on the condition that the resected areas had to be near each other on the MRI scan, even if the ictal epileptic attack revealed by EEG was unable to differentiate the mesial hippocampus and lateral neocortex due to the insufficient depth of the electrodes. When comparing patients with dual pathology lesion resection with patients who had the temporal lesion removed but the hippocampus was spared, an 
improved prognosis was suggested (60.0 vs. $11.1 \%)$. However, the decision of resection of dual pathology remains problematic without sufficient proof that can confine epileptic onset to the ATL (mesial or lateral) or if there were only wide spread epileptic discharges observed in the EEG. The concern for potential postsurgical emotional or memory dysfunctions due to hippocampus resection remains a priority.

In conclusion, in patients with TLE the resection of all lesions had an improved outcome. However, without complete evidence for dual pathology, establishing a surgical strategy may still be possible. Locating the lesions using MRI scans and EEG monitoring may be the key factors in the decision of lesion removal.

\section{Acknowledgements}

Not applicable.

\section{Funding}

No funding was received.

\section{Availability of data and materials}

The datasets used and/or analyzed during the current study are available from the corresponding author on reasonable request.

\section{Authors' contributions}

KY made substantial contributions to conception and design of study. LL collected, analyzed and interpreted patients' data. YQS analyzed patient data and was a major contributor in writing the manuscript. All authors read and approved the final manuscript.

\section{Ethics approval and consent to participate}

This research was approved by the Ethical Committee of The Second Hospital of Dalian Medical School (Liaoning, China). The patients or parents provided written informed consent for the publication of any associated data and accompanying images. No identifying information of patients was included in this manuscript.

\section{Patient consent to publication}

Not applicable.

\section{Competing interests}

The authors declare that they have no competing interests.

\section{References}

1. Stafstrom CE and Carmant L: Seizures and epilepsy: An overview for neuroscientists. Cold Spring Harb Perspect Med 5: a022426, 2015

2. Wiebe S, Blume WT, Girvin JP and Eliasziw M: Effectiveness and Efficiency of Surgery for Temporal Lobe Epilepsy Study Group: A randomized, controlled trial of surgery for temporal-lobe epilepsy. N Engl J Med 345: 311-318, 2001.

3. Harroud A, Bouthillier A, Weil AG and Nguyen DK: Temporal lobe epilepsy surgery failures: A review. Epilepsy Res Treat 2012 $201651,2012$.
4. Engel J Jr, Wiebe S, French J, Sperling M, Williamson P, Spencer D, Gumnit R, Zahn C, Westbrook E and Enos B; Quality Standards Subcommittee of the American Academy of Neurology; American EpilepsySociety; American Association of Neurological Surgeons: Practice parameter: Temporal lobe and localized neocortical resections for epilepsy: Report of the quality standards subcommittee of the american academy of neurology, in association with the american epilepsy society and the american association of neurological surgeons. Neurology 60 : 538-547, 2003.

5. Cendes F, Cook MJ, Watson C, Andermann F, Fish DR, Shorvon SD, Bergin P, Free S, Dubeau F and Arnold DL: Frequency and characteristics of dual pathology in patients with lesional epilepsy. Neurology 45: 2058-2064, 1995.

6. Salanova V, Markand O and Worth R: Temporal lobe epilepsy: Analysis of patients with dual pathology. Acta Neurol Scand 109: 126-131, 2004.

7. Jung R, Aull-Watschinger S, Moser D, Czech T, Baumgartner C Bonelli-Nauer $\mathrm{S}$ and Pataraia E: Is reoperation an option for patients with temporal lobe epilepsy after failure of surgery? Seizure 22: 502-506, 2013.

8. Mathon B, Bielle F, Samson S, Plaisant O, Dupont S, Bertrand A, Miles R, Nguyen-Michel VH, Lambrecq V, Calderon-Garcidueñas AL, et al: Predictive factors of long-term outcomes of surgery for mesial temporal lobe epilepsy associated with hippocampal sclerosis. Epelepsia 58: 1473-1485, 2017.

9. Engel J Jr: Update on surgical treatment of the epilepsies. Clin Exp Neurol 29: 32-48, 1992.

10. Tellez-Zenteno JF, Dhar R and Wiebe S: Long-term seizure outcomes following epilepsy surgery: A systematic review and meta-analysis. Brain 128: 1188-1198, 2005.

11. Tonini C, Beghi E, Berg AT, Bogliun G, Giordano L, Newton RW, Tetto A, Vitelli E, Vitezic D and Wiebe S: Predictors of epilepsy surgery outcome: A meta-analysis. Epilepsy Res 62: 75-87, 2004.

12. Spencer S and Huh L: Outcomes of epilepsy surgery in adults and children. Lancet Neurol 7: 525-537, 2008.

13. Radhakrishnan K, So EL, Silbert PL, Jack CR Jr, Cascino GD, Sharbrough FW and O'Brien PC: Predictors of outcome of anterior temporal lobectomy for intractable epilepsy: A multivariate study. Neurology 51: 465-471, 1998.

14. Jeong SW, Lee SK, Hong KS, Kim KK, Chung CK and Kim H: Prognostic factors for the surgery for mesial temporal lobe epilepsy: Longitudinal analysis. Epilepsia 46: 1273-1279, 2005.

15. Bocti C, Robitaille Y, Diadori P, Lortie A, Mercier C, Bouthillier A and Carmant L: The pathological basis of temporal lobe epilepsy in childhood. Neurology 60: 191-195, 2003.

16. Kelemen A, Barsi P, Eross L, Vajda J, Czirjak S, Borbely C, Rasonyi $\mathrm{G}$ and Halasz P: Long-term outcome after temporal lobe surgery-prediction of late worsening of seizure control. Seizure 15: 49-55, 2006.

17. Louis DN, Perry A, Reifenberger G, von Deimling A, Figarella-Branger D, Cavenee WK, Ohgaki H, Wiestler OD, Kleihues P and Ellison DW: The 2016 world health organization classification of tumors of the central nervous system: A summary. Acta Neuropathol 131: 803-820, 2016.

18. Palmini A, Najm I, Avanzini G, Babb T, Guerrini R, Foldvary-Schaefer N, Jackson G, Luders HO, Prayson R, Spreafico R and Vinters HV: Terminology and classification of the cortical dysplasias. Neurology 62 (Suppl 3): S2-S8, 2004.

19. Eriksson SH, Nordborg C, Rydenhag B and Malmgren K: Parenchymal lesions in pharmacoresistant temporal lobe epilepsy: Dual and multiple pathology. Acta Neurol Scand 112: $151-156,2005$

20. Mueller SG, Laxer KD, Cashdollar N, Lopez RC and Weiner MW: Spectroscopic evidence of hippocampal abnormalities in neocortical epilepsy. Eur J Neurol 13: 256-260, 2006.

21. Parmar H, Lim SH, Tan NC and Lim CC: Acute symptomatic seizures and hippocampus damage: DWI and MRS findings. Neurology 66: 1732-1735, 2006.

22. Briellmann RS, Wellard RM and Jackson GD: Seizure-associated abnormalities in epilepsy: Evidence from MR imaging. Epilepsia 46: 760-766, 2005.

23. Lawn N, Londono A, Sawrie S, Morawetz R, Martin R, Gilliam F, Faught E and Kuzniecky R: Occipitoparietal epilepsy, hippocampal atrophy, and congenital developmental abnormalities. Epilepsia 41: 1546-1553, 2000.

24. Li LM, Cendes F, Andermann F, Watson C, Fish DR, Cook MJ, Dubeau F, Duncan JS, Shorvon SD, Berkovic SF, et al: Surgical outcome in patients with epilepsy and dual pathology. Brain 122: 799-805, 1999. 